Ueber die Einwirkung des Leuchtgases auf verschiedene Salzsolutionen, insbesondere auf eine ammoniakalische Kupferchlorürlösung;

\title{
von Rud. Boettger.
}

(Aus dem nächstens erscheinenden Jahresbericht des physikalischen Vereins in Frankfurt a. M. für 1857 bis 1858 mitgetheilt).

Bei meinen fortgeselzten, bereits im Jahr 1852 begonnenen Versuchen über das Verhalten des gewöhnlichen (aus Steinkohle oder aus Harz oder aus einem Gemenge von Bogheadkohle und harzigem Holz bereiteten) Leuchtgases zu verschiedenen Salzsolutionen und Flüssigkeiten *), sah ich mich, behufs der Entfernung verschiedener, die Leuchtkraft jenes Gases schwächender Beimischungen, veranlafst, unter anderem auch das von Leblanc so aufserordentlich gerühmte Absorbens für Kohlenoxydgas, nämlich eine ammoniakalische Kupferchlorürlösung, in Anwendung zu bringen. Leblanc giebt an $* * *$ ), und Prof. Vog el jun. bestätigt ${ }^{*} * *$ ), dafs wenn man einen Strom Kohlenoxydgas durch eine Auflösung von Kupferchlorür in Chlorwasserstoffsäure, oder durch eine Auflösung von Kupferchlorür in Aetzammoniakflüssigkeit gehen lasse, das Gas mit einer solchen Geschwindigkeit absorbirt werde, wie Kohlensäure durch caustisches Kali, und dafs dadurch ein neues analytisches Mittel gefunden sei, das Kohlenoxydgas mil Leichtigkeit aus Gasgemengen abzuscheiden.

*) Man vergleiche den Jahresbericht des physikalischen Vereins in Frankfurt a. H. 1852 bis 1853.

**) Erdmann's Journal für practische Chemie I, 239 (diese Annalen LXXVI, 278).

***) Dingler's polytechnisches Journal CXXXVI, 237. 
Dieser Angabe vermag ich nicht unbedingt beizupflichten, denn wiewohl einer ammoniakalischen Kupferchlorürlösung, meinen Erfabrungen zufolge, das Vermögen, Kohlenoxydgas zu absorbiren, nicht abgesprochen werden kann, so geschieht diese Absorption doch mit einer solchen Langsamkeit und Trägheit, dafs beim ruhigen Stehen des Gases über genannter Flüssigkeit Stunden vergehen, ehe eine auffallende Einwirkung sich bemerklich macht. Ja, leitet man einen ganz langsamen Strom, durch Kalilösung seiner Kohlensäure völlig beraubten, Kohlenoxydgases (erhalten durch Erhitzen krystallisirter Oxalsäure in concentrirter Schwefelsäure) durch $d r e i$ hinter einander aufgestellte, mit besagter ammoniakalischer Kupferchlorürlösung gefüllte Liebig'sche Kugelröhren, so sieht man, bei noch so lange andauerndem Gasstrome, in der Kupfersalzlösung weder eine Trübung, noch einen Niederschlag entstehen, und das der letzlen Kugelröhre entströmende Gas hei Annäherung einer brennenden Kerze mit der ihm eigenthümlichen, schön blau gefärbten Flamme gerade so ruhig und anhaltend fortbrennen, als ob dasselbe gar kein Absorptionsmittel passirt habe. Da nun überdiels, wie wir sogleich sehen werden, eine ammoniakalische Kupferchlorülösung in einem noch weit höheren Grade von einem im Leuchtgase enthaltenen Kohlenwasserstoffe afficirt, respective zersetzt wird, so dürfte genannter Salzsolution, als vorzugsweisem Absorptionsmittel für Kohlenoxydgas, keineswegs die Bedeutung beizulegen sein, die ihr die Obengenannten vindiciren.

Es erscheint in der That auffallend, dafs Herr Le blanc, der sich in Gemeinschaft mil Prof. Stas und Doyère des ammoniakalischen Kupferchlorürs als Absorbens für Kohlenoxydgas in einem Beleuchtungsgase bediente, Erscheinungen und Reactionen übersehen oder unbeachtet lassen konnte, die hierbei schon nach Verlauf von wenigen Minuten unfehl- 
bar in einem besonders auffallenden Grade hervorzutreten plegen. Ueberhaupt finde ich dieser merkwürdigen Erscheinungen, welche sich bei der Einwirkung von Leuchtgas auf eine ammoniakalische Kupferchlorürlösung kund geben, und wovon hier sogleich ausführlicher die Rede sein wird, in der chemischen Literatur mit keiner Sylbe Erwähnung gethan *).

Leitet man nämlich gewöhnliches Leuchtgas, ehe man es in die gegenwärtig fast allgemein in den Laboratorien eingeführten Bunsen'schen Gaslämpchen einströmen läfst, durch eine, zu $\% \frac{3}{3}$ mit ammoniakalischer Kupferchlorïrlösung **) gefüllte, ungefähr 12 bis 16 Cubikzoll Rauminhalt fassende Zwischenflasche, der Art, dafs dasselbe in diese durch eine in dem durchbohrten Korke befestigte, einige Linien unterhalb des Niveau's der Flüssigkeit ausmündende Glasröhre eintritt und durch eine zweite knieförmig gebogene, dicht unter dem Kork der Flasche sich endigende, durch eine Caoutchoucröhre mit dem Gaslämpchen communicirende Glasröhre austritt, so sieht man schon nach Verlauf von wenigen Minuten die oberen Innenwände dieser $\mathrm{Z}_{\text {wischenflasche sich mit einem }}$ fast zinnoberrothen Anfluge überzichen; nach und nach erfült sich auch die blaue Flüssigkeil mit einer Menge zinnoberroth gefärbter Flocken, die nach Verlauf von wenigen Stunden sich so bedeutend anhäufen, dafs sie nicht selten das ganze untere Viertel der Flasche einnehmen. Durch Mitanwendung einer oder mehrerer solcher Wasch- oder Zwischenflaschen, die man mit den verschiedenartigsten Salzsolutionen füllen kann, hat

*) Ueber solche explosive Kupfer- und Silberverbindungen, mit gasförmigen Zersetzungsproducten des Alkohols dargestellt, vgl. diese Annalen CVIII, $116 \mathrm{f}$.

D. $R$.

**) Ein wenig beigemengtes Kupferchlorid-Ammoniak wirkt nicht störend ein.

Anmal. d, Chom. a. Pharm. CIx. Bd. 3. Helt. 
man sonach eine einfache Vorrichtung, mit welcher sich ohne besonderen Gasalwand die mannigfalligsten Absorptionsversuche mit brembaron Gasen aller Art anstellen lassen, indem hierbei das Gaslämpchen gleichzeitig nebenbei zum Erhizen und Abdampfen von Flüssigkeiten u. s. w. wie gewöhnlich benutal werden kann.

Da weder bei anhaltendem Hindurchleiten von Kohlenoxydgas, wie oben erwähnt, noch auch beim Hindurchleiten von reinem Wasserstoffgas, defsgleichen von kohlensaurem Gas durch die ammoniakalische Kupferchlorürlösung eine sichtbare Veränderung in dieser letzteren eintritt, so lag die Vermuthung nahe, es möchte, da, wie wir nachher sehen werden, allem Anscheine nach jener rothe flockige Körper eine Kupfer-Kohlenwasserstoffverbindung ist, vielleicht das in dem gewöhnlichen Leuchtgase in nicht unbedeutender Menge enthattene Grubengas $\left(\mathrm{C}_{2} \mathrm{H}_{4}\right)$ oder das Elaylgas $\left(\mathrm{C}_{4} \mathrm{H}_{4}\right)$ die Veranlassung zur Enistehung jenes flockigen zinnoberrothen Körpers geben. Directe, mit den genannten Gasen angestellte Versuche ergaben aber ein ganz unzweideuliges negatives Resultal. Auch durch Schülteln von im Leuchtgase nicht selten suspendirtem Benzol $\left(\mathrm{C}_{12} \mathrm{H}_{6}\right)$ und Naphtalin $\left(\mathrm{C}_{20} \mathrm{H}_{8}\right)$ mit der ammoniakalischen Kupfersolution vermochte ich nicht, den rothen Körper zum Vorschein zu bringen. Es blieb daher nur noch übrig, die ammoniakalische Kupferchlorürlösung auch mit den übrigen, im Leuchtgase gleichfalls nichl selten in wechselnden Mengen vorkommenden höheren Kohlenwasserstoffen in Wechselwirkung treten zu lassen, namentlich mit Propylen $\left(\mathrm{C}_{6} \mathrm{H}_{6}\right)$, Butylen $\left(\mathrm{C}_{8} \mathrm{H}_{8}\right)$ und Amylen $\left(\mathrm{C}_{10} \mathrm{H}_{10}\right)$. Das bei der trockenen Destillation von gleichen Gewichtsheilen wasserfreiem essigsanrem Natron und Natronkalk auftretende Gasgemenge, worin bekannllich alle die Ietztgenannten Kohlenwasserstoffe enthalten sind, fand ich indefs ohne alle Einwirkung anf Kupferchlorür-Ammoniak. 
Was für ein Kohlenwasserstoff nun aber mil dem Kupfer verbunden jenen merkwürdigen, mil den auffallendsten Eigenschaften begabten Körper repräsentirt, das wird erst dann mit Bestimmtheit ausgesprochen werden können, wenn, was mir bis jelzt noch nicht hat gelingen wollen, Verfahrungsweisen zu seiner Entstehung werden ausfindig gemacht werden sein, die, ihn von der zu einer quantitativen Analyse erforderlichen Reinheit zu erhalten, geeignet sein werden. Versucht man nämlich, ihn (was ich noch am zweckmälsigsten gefunden) auf einem Filter mit etwas Aetzammoniakflüssigkeit und hierauf mil Alkohol auszusüfsen, so erkennt man schon an der sehr bald eintretenden Veränderung seiner Farbe, die namentlich bei seinem Trocknen von Zinnoberroth in Bräunlichviolett übergeht, seine grofse Unbeständigkeit. So viel geht aber schon jelzl aus den von mir angestellten qualitativ analytischen Versuchen hervor, dafs wir es hier mit einem Körper von ganz ungewöhnlicher Zusammensetzung zu thun haben, in welchem der Kohlenwasserstoff als zusammengesetztes Radical, ähnlich dem Cyan, mit dem Kupfer in Function trilt.

Auch mit dem Golde und mit dem Silber ist es mir gelungen, analoge Verbindungen, deren ich nachher in der Kürze Erwähnung thun werde, hervorzubringen.- Leitet man anhaltend einen Strom Leuchtgas durch eine Auflösung von Kupferchlorür in Chlorwasserstoffsäure, so nimmt man in dieser Flüssigkeit keine Veränderung wahr; dagegen sieht man den rothen Körper bei gleicher Behandlung in kurzer Zeit sich bilden in einer ammoniakalischen Lösung des canariengelben unterschwefligsauren Kupferoxydulnatrons (erhallen durch Fällung einer Kupfervitriollösung mittelst unterschwefligsauren Natrons), so wie in einer ammoniakalischen Lösung des orangefarbenen schwefligsauren Kupferoxyduloxyds (erhalten 
durch Erhitzen einer Kupfervitriollösung mit schwefligsaurem Natron).

In Bezug auf die Eigenschaften dieses interessanten Körpers erlaube ich mir Folgendes von denselben hier anzuliihren:

Im feuchten Zristande erscheint der Körper flockig, nicht krystallinisch, fast zinnoberroth; im trockenen Zustande sieht er braun aus, mit einem Stich ins Violette. Staubtrocken zwischen Fliefspapier gelegt und auf einem eisernen Ambos mit einem eisernen Hammer mälsig stark geschlagen, zersetzt er sich unter Funkensprühen und zischendem Geräusch, mit finterlassung einer grofsen Menge eines sammetschwarzen, ungemein voluminösen Pulvers (bestehend aus fein zertheilter Kohle und metallischem Kupfer). In einem dünnwandigen Reagensglase erhitzt zersetzt er sich, je nachdem er plötzlich oder langsam ansteigend erhitzt wird, zwischen 95 und $120^{\circ}$ R., unler ziemlich starker Verpuffung, mit gleichzeitiger Hinterlassung eines, wenigstens das Dreifache seines ursprünglichen Volumens betragenden, sammetschwarzen Pulvers (eines Gemisches von Kohle und Kupfer). Im feuchten Zustande wird er bei mittlerer Temperatur von sehr verdünnter Salzsäure, beim Schülteln ohne Zersetzung, d. h. ohne Gasentwickelung, zu einer grünlichgelb gefärbten Flüssigkeit gelöst, die ohne sich zu verändern mit einer willkürlich groisen Menge Wassers versetzt werden kann, und aus welcher, bei unvollständiger Neutralisation mittelst causlischem Kali, der Körper mit seiner ursprünglichen rothen Farbe und mit allen seinen ursprünglichen Eigenschaften gefällt wird. Behandelt man ihn dagegen im feuchten Zustande bei miltlerer Temperatur mil Salzsäure von 1,1 spec. Gew., so löst er sich unter kaum merklicher und sehr bald nachlassender Gasentwickelung theilweise darin auf; aber bei Anwendung von Hitze erfolgt eine vollständige Zersetzung desselben, unter sehr tumultuarischer Gasentwickelung; das dabei auf- 
tretende Gas ist entzündlich and brennt bei Annäherung einer Kerze, unter gleichzeitiger Rul'sabsonderung, mit einer intensiv gelblichweils gefärbten Flamme. Die bei diesem Vorgange resultirende, ihres Gasgehaltes beraubte Flüssigkeit besieht lediglich aus salzsäurehaltigem Kupferchlorür, aus welchem Aetzkali gelbes Kupferoxydulhydrat fält. Von Essigsäure, verdünter Schwefelsäure, Kali-, Natron- und Ammoniaklösung wird der rothe Körper, selbst in der Wärme, nicht angegriffen, dagegen von einer concentrirten Lösung von Cyankalium schon bei initterer Temperatur, unter Kohlenwasserstoffgasentwickelung, zu einer ungefärbten Flüssigkeit aufgelöst, aus welcher beim Abdampfen leichtlösliches Kaliumkupfercyanür in Krystallen gewonnen werden kann. Wirft man etwas von dem staubtrockenen Körper in eine mit Chlorgas gefüllte Flasche, so erfolgt augenblicklich, unter Lichtentwickelung, eine schwache Detonation, es bildet sich Chlorkupfer, Salzsäure, und fein zertheilte Kohle scheidet sich ab. Wickelt man ein wenig von dem staubtrockenen Pulver in das eine Ende eines langen Streifens Flielspapier locker ein und senkt diesen Streifen dann in eine Atmosphäre von Chlor, so findet gleichfalls fast momentan eine gefahrlose schwache Verpuffung statt, während man in dem nicht selten ganz unverletzt bleibenden Papierstreifen bei dessen Aufwickeln eine Menge" überaus voluminöser Kohle antrifft. Auch beim Einsenken des locker in Fliefspapier eingewickelten Pulvers in eine Atmosphäre von Bromdampf findet Entzïndung, respective Zersetzung desselben, unter gleichzeitiger Ausscheidung von fein zertheilter Kohle, statt. Mengt man nahezu gleiche Raumtheile des staubtrockenen explosiven Körpers mit fein gepulvertem Jod, und zwar unter Vermeidung jedweder Friction, so erfolgt in wenig Augenblicken, unter zischendem Geräusch, eine Entzündung des Gemisches, mit Hinterlassung von fein zertheilter Kohle. Mengt man ihn mit 
einem gleichen Raumtheile chlorigsauren Bleioxyds recht innig, so reicht eine ganz unbedeutende Friction hin, das Gemisch zum Explodiren zu bringen. Hatte der unter einer Glasglocke über Schwefelsäure getrocknete explosive Körper sich während dieses Trocknens vielleicht ein wenig oxydirt, so zeigt er nicht mehr die Eigenschaft, mit gepulvertem Jod gemischt sich von selbst unter Zischen za zersetzen; um ihn aber hierzu tauglich zu machen, braucht man ihn nur einige Male mit Aetzammoniakfliussigkeit zu digeriren, dann mit Alkohol auszusüfsen und in einer Atmosphäre von Wasserstoffgase zu trocknen.

Aus den hier angeführten Reactionen ist man nun wohl schon jetzt berechtigt, den in Rede stehenden explosiven Körper für ein Kupferkohlenhydrür zu halten, das sich in gewisser Beziehung dem von Wurtz*) entdeckten, bei der Aufeinanderwirkung von Kupfervitriollösung und unterphosphoriger Säure sich bildenden, und auch dem von Pogg endorff**) auf electrischem Wege, bei der Zerlegung einer schwach gesäuerten und hinreichend verdünnten Kupfervitriollösung mittelst eines mälsig stark wirkenden galvanischen Stromes an der Kathode gewonnenen Kupferhydrür anreihen lassen dürfte. Jedenfalls werde ich mir angelegen sein lassen, eine geeignetere Bereitungsweise dieses interessanten Körpers, als die bisher von mir befolgte und hier beschriebene, ausfindig zu machen, damit derselhe in einem reineren und der Zersetzung weniger unterworfenen Zustande einer quantitativen Analyse unterworfen werden könne.

Was schliefslich die Einwirkung des Leuchtgases auf gewisse Gold- und Silbersolutionen betrifft, so habe ich hierüber zur Zeit nur erst einige wenige Versuche angestell,

*) Man vergleiche Pogg. Ann. LXIII, 476 (diese Annalen LII, 256).

*) Pogg. Ann. LXXV, 350 . 
die ich jedoch, trotz ihrer Unvollständigkeit, hier noch folgen lassen will.

Leitet man anhaltend gewöhnliches Leuchtgas durch eine Auflösung von salpetersaurem Silberoxydammoniak mit vorwaltendem Ammoniak, so bemerkt man schon nach Verlauf von wenigen Minuten die genannte Salzsolution sich schwach röthen und nach und nach einen überaus feinen, schwärzlich grauen, flockigen (nicht krystallinischen) Körper sich darin ausscheiden, dessen Farbe allmälig immer dunkeler, zuletzt ganz schwarz wird. Im völlig getrockneten Zustande zeigt dieser Körper folgende Eigenschaften : Von reiner Salpetersäure von 1,3 spec. Gewicht wird er bei mittlerer Temperatur nicht merklich angegriffen oder verändert. $\mathrm{Er}$ explodirt sowohl durch einen Schlag von Eisen auf Eisen, wie durch Temperaturerhöhung, und zwar mit weit grö/serer Heftigkeit, als die vorhin erwähnte Kupferverbindung. Zersetzt man ihn in ganz kleinen Quantitäten durch Erhitzen in einem etwas langen Reagensglase, so sieht man nach jedesmaliger Verpuffung ein überaus lockeres, ungemein voluminöses, sammetschwarzes (aus fein zertheilter Kohle und Silher bestehendes) Pulver resultiren, das beim Erhitzen auf einem Plalinbleche, bei Zutritt der Luft, unter Hinterlassung metallischen Silbers verglimmt. Wirft man ein kleines Messerspitzchen voll davon in eine mil Chlor gefüllte Flasche, so erfolgt augenblicklich eine starke, immerhin aber völlig gefahrlose Detonation, unter Ausscheidung von Chlorsilber und ungemein voluminöser sammetschwarzer Kohle. Beim Vermischen ungefähr gleicher Raumtheile dieses Stoffes mit fein zerriebenem Jod erfolgt, ohne dafs man die geringste Reibang anzuwenden braucht, fast augenblicklich eine Detonation, wobei wiederum eine grofse Menge fein zertheilter Kohle abgeschieden wird. Ein Gemisch von gleichen Raumtheilen dieser 
explosiven Verbindung und chlorigsaurem Bleioxyd delonirt schon bei der allerleisesten Friction mit furchtbarem Knall.

Beim Hindurchleiten von Leuchtgas durch eine ammoniakalische Lösung von frisch gefälltem Chlorsilber erhält man die explodirende schwarze Silberverbindung nicht. Auch der jüngst von Prof. Vog el ${ }^{*}$ ) beim Hindurchleiten von Leuchtgas durch eine Auflösung von salpetersaurem Silberoxyd cutstehende, mehr grauweifs aussehende krystallinische Niederschlag, der sowohl beim Erhitzen, wie meinen Beobachtungyen zufolge auch beim Eintragen in eine Almosphäre von Cblor mit grofser Heftigkeil explodirt, scheint verschieden zu sein von dem hier in Rede stehenden flockigen schwarz aussehenden Körper.

Auch bei mehrstündigem Hindurchleiten von mittelst verdünnter Schwefelsüure gewaschenem (d. h. ammoniakfreiem) Leuchtgas durch eine möglichst säurefreie Goldchloridlösung, sieht man nach und nach einen bräunlichen Niederschlag sich bilden, der wohl ausgesüfst und getrocknet beim Erhitzen überaus heftig explodirt. Das Glas, in welchem die Zersetzung der Goldsalzsolution durch das Leuchtgas vor sich geht, pflegt sich hierbei im Inneren mit einem ganz dünnen, fest haftenden, überaus glänzenden Goldhäutchen zu überziehen.

Nachdem vorstehende Zeilen bereits fertig zum Druck niedergeschrieben waren, habe ich die Freude gehabt, noch eine liupferverbindung ausfindig zu machen, die im hohen Grade geeignet zu sein scheinl, bei ihrer Zersetzung mittelst Leuchtgas das explosive Kupferkohlenhydrür von einer solchen Reinheit und Beständigkeil zu liefern, wie diels zu einer Analyse nothwendig ist; ich werde mir daher angelegen sein lassen, über die chemische Constitution dieses bis jetzt

*) Siehe Bayerisches Kunst- u. Gewerbeblatt 1858, S. 26. 
ganz einzig dastehenden räthselhaften Körpers späterhin ausführlicher zu berichten, und führe hier vorläufig nur so viel an, dafs man beim Hindurchleiten von Leuchtgas durch eine Kupferoxydul-Ammoniallösung *) den mehrerwähnten Körper gleich bei seinem Entstehen in Gestalt eines dunkel-violettroth gefärbten flockigen Niederschlags hervorgehen sieht, der nur mit W'asser ausgesüfst zu werden braucht, um ihn von hinlänglicher Reinheit zu erhalten. Sein äulseres Ansehen verändert sich selbst während des Trocknens nicht wesentlich, und er scheint die mehrfach erwähnten explosiven Eigenschaften noch in einem etwas stärkeren Grade zu besitzen, als das aus Kupferchlorür-Ammoniak gewonnene l'räparat. - Die blaue Flüssigkeit, welche man bei anhallendem Schütteln von Kupferasche mit starker Actzammoniakflüssigkeit erhäl, besteht fast nur aus Kupferoxydulammoniak, mit einer äufserst geringen Beimischung von hupferoxydammoniak; beim Hindurchleiten von Leuchtgas entfärbt sie sich schon in wenig Viertelstunden vollständig, und setzt man dann noch ferner das Hindurchleiten von Leuchtgas fort, so gewinnt man in verhältnifsmälsig kurzer Zeit sehr beträchtliche Quantiläten jenes interessanten Körpers.

Dieser Tage ist mir auch gelungen, durch doppelte Wahlverwandtschaft das Kupfer in der explosiven Kupferkohlenwasserstoffverbindung durch Silber zu substituiren; mau braucht nämlich jene Kupferverbindung im noch feuchten Zustande nur mit einer Auflösung von Höllenstein bei mittlerer Temperatur zu überschütten und das Gemisch einige Male umzurühren; es bildet sich hierbei augenblicklich das

*) Erhalten durch längeres Schütteln von fein zerriebener staubförmiger Kupferasche (bekanntlich cinem Gemisch von Kupferoxydul, Kupferoxyd und metallischem liupfer) mit starker Aetzammoniakflüssigkeit in einer nicht ganz damit gefüllten, aber wohl verschlossenen dickwandigen Glasflasche. 
schwarze Silberkohlenhydrür, während salpetersaures Kupferoxyd gelöst bleibt. Das auf diese Weise gewonnene Präparat ist identisch mit dem direct aus salpetersaurem Silberoxydammoniak und Leuchlgas gewonnenen.

Auf diesem hier zuletzt angedeuteten Wege wird es vielleicht möglich sein, noch manche andere Metalle mit jenem Kohlenwasserstoff, dessen chemische Constitution, wie gesagt, noch zu ermilteln ist, in Verbindung treten zu lassen.

Ueber eine neue, aus Dreifach-Bromallyl und Ammoniak entstehende Base; von M. Simpson*).

Das von $W$ urt $z *$ ) in neuerer Zeit durch Behandlung von Jodallyl mit überschüssigem Brom dargestellte DreifachBromallyl $\mathrm{C}_{6} \mathrm{H}_{5} \mathrm{Br}_{3}$ wird durch Ammoniak leicht angegriffen und bildet mit ihm eine neue ölige Base, das Dibromallylamin.

Zur Darstellung des salzsauren Salzes dieser Base wird 1 Vol. Dreifach-Bromallyl mit etwa 6 Volumen einer Lösung von Ammoniak in schwachem Alkohol gemischt, die Mischung in zugeschmolzenen Glasröhren während 10 bis 12 Stunden auf $100^{\circ}$ erhitzt, wo sich Bromammonium ausscheidet, dann nach dem Oeffnen der Röhren die Flüssigkeit vom Bromammonium abfiltrirt und das Filtrat mit viel Wasser versetzt, wo sofort Trübung und allmälig Ausscheidung eines schweren Oeles eintritt. Dieses Oel (die neue, noch mit etwas un-

*) Aus Phil. Mag. [4] XVI, 257.

**) Diese Annalen CIV, 247 .

D. $\boldsymbol{R}$. 